University of New Orleans

ScholarWorks@UNO

Physics Faculty Publications

Department of Physics

2005

\title{
Magneto-optical spectra of closely spaced magnetite nanoparticles
}

Damon Allen Smith

Yu A. Barnakov

B L. Scott

S A. White

Kevin L. Stokes

University of New Orleans

Follow this and additional works at: https://scholarworks.uno.edu/phys_facpubs

Part of the Nanoscience and Nanotechnology Commons, and the Physics Commons

\section{Recommended Citation}

J. Appl. Phys. 97, 10M504 (2005)

This Article is brought to you for free and open access by the Department of Physics at ScholarWorks@UNO. It has been accepted for inclusion in Physics Faculty Publications by an authorized administrator of ScholarWorks@UNO.

For more information, please contact scholarworks@uno.edu. 


\title{
Magneto-optical spectra of closely spaced magnetite nanoparticles
}

\author{
Damon Allen Smith, Yu. A. Barnakov, B. L. Scott, S. A. White, ${ }^{\text {a) }}$ and K. L. Stokes ${ }^{\text {b) }}$ \\ Department of Physics and Advanced Materials Research Institute, University of New Orleans, New Orleans, \\ Louisiana 70148
}

(Presented on 10 November 2004; published online 16 May 2005)

\begin{abstract}
The Faraday rotation spectrum of composites containing magnetite nanoparticles is found to be dependent on the interparticle spacing of the constituent nanoparticles. The composite materials are prepared by combining chemically synthesized $\mathrm{Fe}_{3} \mathrm{O}_{4}$ (magnetite) nanoparticles (8-nm diameter) and poly(methylmethacrylate). Composites are made containing a range of nanoparticle concentrations. The peak of the main spectral feature depends on nanoparticle concentration; this peak is observed to shift from approximately $470 \mathrm{~nm}$ for (dilute composites) to $540 \mathrm{~nm}$ (concentrated). We present a theory based on the discrete-dipole approximation which accounts for optical coupling between magnetite particles. Qualitative correlations between theoretical calculations and experimental data suggest that the shifts in spectral peak position depend on both interparticle distance and geometrical configuration. (C) 2005 American Institute of Physics.
\end{abstract}

[DOI: $10.1063 / 1.1847352$ ]

\section{INTRODUCTION}

The coupling of light with structures much smaller than the light's wavelength (typically, interaction lengths less than $100 \mathrm{~nm}$ ) is particularly relevant with the current push to fabricate photonic devices on the nanometer scale. ${ }^{1}$ Previous studies of these optical near-field interactions have focused mostly on noble-metal nanoparticles which display resonances in the optical-absorption spectrum dependent on the particle size, shape, and interparticle spacing. ${ }^{2}$ In addition, light has been shown to propagate along noble-metal nanoparticle chains. ${ }^{3}$ Here, we extend the investigations of nearfield optical interactions and include a study of the magnetooptical properties of nanoparticles as a function of interparticle spacing. Magneto-optically active nanoparticles could have applications in the emerging field of nanophotonics, data storage, or sensing.

The magneto-optical properties of films containing dilute concentrations of $\mathrm{Fe}_{3} \mathrm{O}_{4}$ (magnetite) particles have previously been studied. ${ }^{4,5}$ In fact, we recently reported that shifts in the Faraday rotation spectrum of magnetite/polymer nanocomposites are observed with changes in the nanoparticle size, with particle diameters ranging from 8 to $200 \mathrm{~nm} .{ }^{6}$ In this paper, we report on shifts in Faraday rotation peak position dependent on the concentration of nanoparticles in the matrix. We estimate that the magnetite particles are separated (on average) by distances as small as a few nanometers. To explain these results, we present a new theory based on the discrete-dipole approximation which accounts for optical dipole-dipole coupling between the magnetite particles. Although our model is currently limited to two interacting spheres, we can gain a qualitative understanding of the origin of the spectral shifts.

\footnotetext{
a)Also at Dillard University, New Orleans, LA 70122.

b) Author to whom correspondence should be addressed; electronic mail: klstokes@uno.edu
}

\section{THEORY}

The nanocomposite materials are modeled using a modification of the discrete-dipole approximation (DDA). ${ }^{7}$ In our calculation, each particle is approximated by a dipole located at the point $\overrightarrow{\mathbf{r}}_{j}$ with polarizability tensor $\widetilde{\alpha}_{j}$. The polarization of the dipole is given by $\overrightarrow{\mathbf{P}}_{j}=\widetilde{\alpha}_{j} \overrightarrow{\mathbf{E}}_{j}$, where $\overrightarrow{\mathbf{E}}_{j}$ is the local electric field at point $\overrightarrow{\mathbf{r}}_{j}$. When exposed to light, this electric field is the sum of the incident field and the field emitted by all neighboring dipoles ${ }^{8}$

$$
\overrightarrow{\mathbf{E}}_{\mathrm{dipole}, j}=-\sum_{k \neq j} \widetilde{A}_{j k} \overrightarrow{\mathbf{P}}_{k}
$$

where

$$
\tilde{A}_{j k}=\frac{e^{i \kappa r_{j k}}}{r_{j k}}\left[\kappa^{2}\left(\hat{r}_{j k} \hat{r}_{j k}-\tilde{1}_{3}\right)+\frac{i \kappa r_{j k}-1}{r_{j k}^{2}}\left(3 \hat{r}_{j k} \hat{r}_{j k}-\tilde{1}_{3}\right)\right],
$$

and $r_{j k}$ is the distance from particle $j$ to particle $k$ and $\hat{r}_{j k}$ is the unit vector in the $r_{j k}$ direction with $\kappa=\omega / c$. The $3 \times 3$ identity matrix is represented by $\tilde{1}_{3}$. For $N$ particles, the polarizations, of each particle $\overrightarrow{\mathbf{P}}_{j}$ can then be determined by solving the system of $3 N$ complex linear equations

$$
\sum_{k=1}^{N} \widetilde{A}_{j k} \overrightarrow{\mathbf{P}}_{k}=\overrightarrow{\mathbf{E}}_{\mathrm{inc}, j},
$$

where

$$
\tilde{A}_{j j}=\tilde{\alpha}_{j}^{-1} \text {. }
$$

The difficulty in using the existing DDA methods to model magneto-optical materials lies in the presence of antisymmetric off-diagonal components in the dielectric tensor. For a magnetic field applied parallel to the propagation direction of incident radiation, the dielectric tensor takes the form $^{9}$ 


$$
\widetilde{\varepsilon}=\left(\begin{array}{ccc}
\varepsilon_{x x} & i \varepsilon_{x y} & 0 \\
-i \varepsilon_{x y} & \varepsilon_{x x} & 0 \\
0 & 0 & \varepsilon_{x x}
\end{array}\right) .
$$

In order to adapt the DDA method to magneto-optical materials, we transform the dielectric tensor to the right-left $(r l)$ circularly polarized coordinate system with the usual transformation $^{10}$

$$
\widetilde{\varepsilon}^{r l}=\tilde{f}^{-1} \widetilde{\varepsilon} \tilde{f},
$$

where

$$
\tilde{f}=\frac{1}{\sqrt{2}}\left(\begin{array}{ccc}
1 & 1 & 0 \\
-i & i & 0 \\
0 & 0 & \sqrt{2}
\end{array}\right) .
$$

This allows us to use the Classius-Mossotti principle to find the polarizability of the sphere in this coordinate system ${ }^{11}$

$$
\alpha_{j, u}^{r l}=a^{3} \frac{\varepsilon_{j, u}^{r l}-\varepsilon_{\mathrm{hst}}}{\varepsilon_{j, u}^{r l}+2 \varepsilon_{\mathrm{hst}}}
$$

where $a$ is the radius of the spherical particle, $u$ is the diagonalized component (right, left, and $z$ ), and $\varepsilon_{\mathrm{hst}}$ is the dielectric constant of the host medium. Transforming the matrix $\tilde{A}_{j k}$ and the vectors $\overrightarrow{\mathbf{P}}_{k}$ and $\overrightarrow{\mathbf{E}}_{\mathrm{inc}, j}$ into the circular coordinate system with Eq. (7) yields

$$
\sum_{k=1}^{N} \tilde{A}_{j k}^{r l} \overrightarrow{\mathbf{P}}_{k}^{r l}=\overrightarrow{\mathbf{E}}_{\mathrm{inc}, j}^{r l}
$$

with $\widetilde{A}_{j j}^{r l}=\left(\widetilde{\alpha}_{j}^{r l}\right)^{-1}$. Solving Eq. (9) for the polarizations $\overrightarrow{\mathbf{P}}_{k}^{r l}$, an effective polarizability is determined from a volume average of the calculated polarizations. The Faraday rotation spectra $^{12}$ were calculated from the average dielectric components which were found by using the inverse of the ClassiusMossotti relation and the averaged polarizability.

In our calculations, we used literature values for diagonal components of the dielectric tensor of $\mathrm{Fe}_{3} \mathrm{O}_{4}$ in Eq. (5) taken from the data reported by Schlegel et al. ${ }^{13}$ and offdiagonal components taken from Zhang et al. ${ }^{14} \mathrm{~A}$ dielectric constant of 2.25 was used for the poly (methylmethacrylate) (PMMA) $\left(\varepsilon_{\mathrm{hst}}\right)$.

\section{EXPERIMENT}

Magnetite nanoparticles were synthesized using the method of Caruntu et al. ${ }^{15}$ Briefly, $\mathrm{FeCl}_{3}$ and $\mathrm{FeCl}_{2}$ were dissolved in diethylene glycol yielding a solution containing $\mathrm{Fe}^{3+}$ and $\mathrm{Fe}^{2+}$ ions in the ratio of $2: 1$. Coprecipitation with $\mathrm{NaOH}$ at $\sim 200{ }^{\circ} \mathrm{C}$ resulted in $8-10-\mathrm{nm} \mathrm{Fe}_{3} \mathrm{O}_{4}$ particles. The nanoparticles were washed, dried, and suspended in toluene by the addition of oleic acid as a capping ligand. The concentration of this solution was approximately $5 \mathrm{mg} / \mathrm{ml}$. Varying amounts of this nanoparticle solution were then mixed with a $10 \%$ solution of PMMA and toluene. Thin films were prepared by spin coating $20 \mu \mathrm{l}$ of the solution onto quartz disks for $9 \mathrm{~min}$ at $150 \mathrm{rpm}$. Spectral Faraday

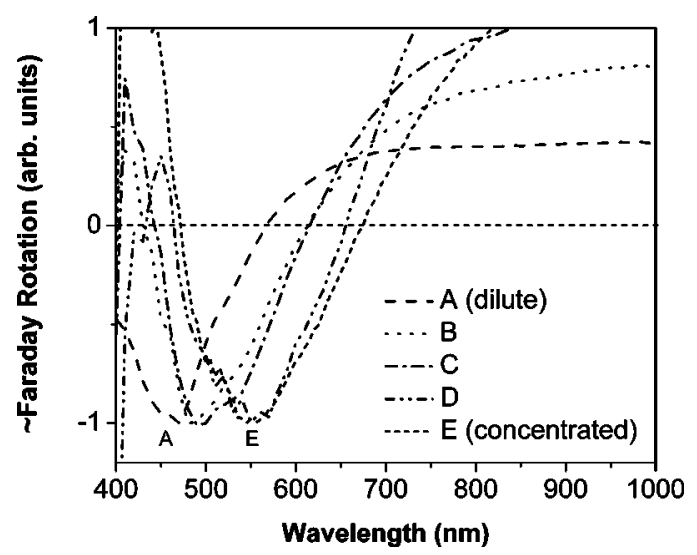

FIG. 1. Experimental measurements of the Faraday rotation of an $\mathrm{Fe}_{3} \mathrm{O}_{4}$ nanoparticle/polymer composite film. The polymer is loaded with an increasing concentration $(A \rightarrow E)$ of 8 -nm particles. The primary peak of the spectral Faraday rotation for concentrations $A$ and $E$ is labeled on the graph.

rotation measurements were made in the polar configuration using the photoelastic modulator technique ${ }^{16}$ as described in Ref. 6.

\section{RESULTS AND DISCUSSION}

Figure 1 shows the Faraday rotation spectra of magnetite nanoparticle/PMMA composite films. By increasing the concentration of magnetite in the composite materials, shifts in the main spectral feature of the Faraday rotations by up to approximately $70 \mathrm{~nm}$ are observed. Films produced with dilute concentrations exhibited peaks at approximately 470 $\mathrm{nm}$, while those at higher concentrations shifted towards red wavelengths. The largest shift was observed for the most concentrated composite at approximately $540 \mathrm{~nm}$.

These shifts in the spectral peak are not predicted by effective-medium theory (EMT) which does not account for optical interactions between the particles. ${ }^{12}$ We use our modified DDA model to explicitly account for particleparticle interactions. Here, we limit the calculations to two coupled $\mathrm{Fe}_{3} \mathrm{O}_{4}$ nanoparticles and find that this is sufficient to reproduce the qualitative features of the measured Faraday rotation spectrum. We calculate the spectrum for the three basic two-particle geometries shown in Figs. 2-4. In these calculations, the magnetic field is parallel to the direction of light propagation, taken to be the $z$ direction, consistent with Eq. (5) and the polarization of the incident light is in the $x$ direction.

For all particle geometries, little change was seen in the spectra until the particles were brought within approximately 7 or $8 \mathrm{~nm}$ of each other. In fact, for interparticle distances greater than $8 \mathrm{~nm}$, our calculation is similar to EMT, which is included in the figures for comparison. Figure 2 shows the theoretical results for two particles aligned on the $x$ axis perpendicular to the propagation direction and parallel to the linear polarized light. This geometry shows a progressive redshift to approximately $500 \mathrm{~nm}$ for dipoles with a $3-\mathrm{nm}$ spacing. In Fig. 3, the particles are, again, aligned perpendicular to the propagation direction but with the incident light polarized perpendicular to the particles. Redshifts are shown with a maximum at around $510 \mathrm{~nm}$, but little differ- 


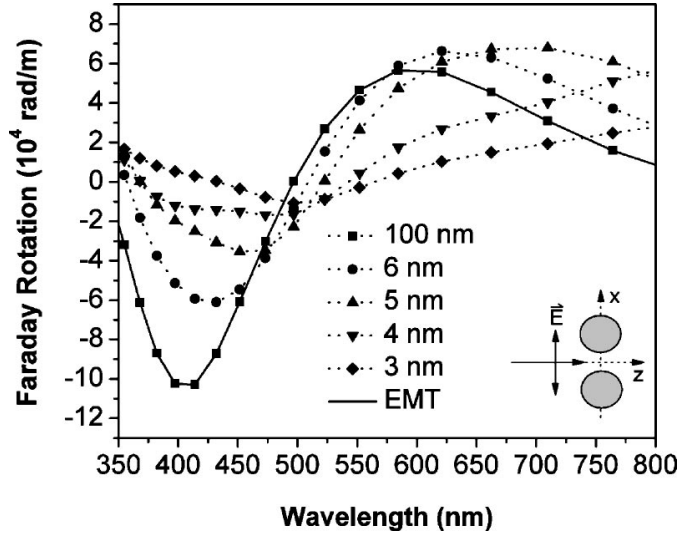

FIG. 2. Calculation of the spectral Faraday rotation for two closely spaced 8 -nm $\mathrm{Fe}_{3} \mathrm{O}_{4}$ spheres. The direction of the propagation and the polarization of the optical field are indicated in the inset. The EMT calculation (solid line) overlaps completely with the modified DDA calculation for $100-\mathrm{nm}$ dipole separation (solid squares).

ence is seen in the spectra until the particles are within $4 \mathrm{~nm}$. In the last geometry, shown in Fig. 4, the particles are aligned parallel to the propagation direction. In contrast with the other geometries, blueshifting is observed in these calculations down to less than $475 \mathrm{~nm}$ at a 3-nm spacing. We point out that if we assume we have 8-nm-diameter particles, separations less than $8 \mathrm{~nm}$ would correspond to particles which physically overlap. This is justified within this approximation since the physical particle is replaced by a radiating dipole at the particle's position. Size is only taken into account in calculating the "strength" of the polarizability [Eq. (8)]. Phenomenologically, this overlap accounts for multipolar corrections to the depolarization factor. ${ }^{17}$ In the experimental system, the particles are expected to be randomly distributed with an average interparticle spacing determined by the volume fraction of nanoparticles to PMMA host material. The measured spectrum would then be an average of the three basic particle geometries of Figs. 2-4. Interestingly, our calculations show that the primary features of the Faraday rotation, that is, a redshift and broadening of the main peak, can be qualitatively explained by near-field optical interactions between two particles. As in previous work on optical scattering by clusters of noble-metal nanoparticles, the main features of the spectra are described by a two-particle approxi-

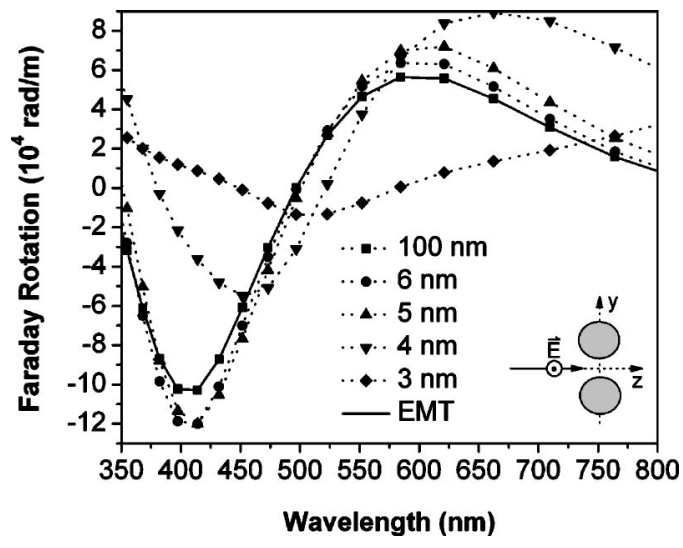

FIG. 3. Calculation of the spectral Faraday rotation for the polarization direction perpendicular to the two-sphere axis.

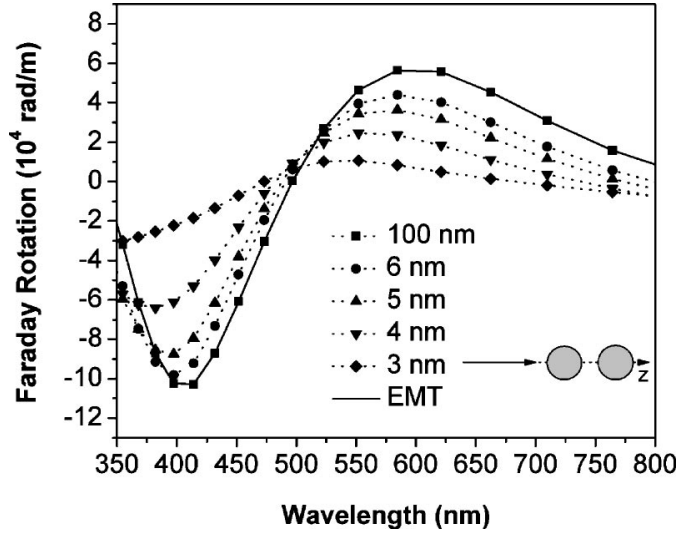

FIG. 4. Calculation of the spectral Faraday rotation for light propagating along the axis of the two-sphere system.

mation. Because the optical coupling is due to near-field interactions, the polarizability of a nanoparticle is determined largely by the contributions from its nearest neighbors. ${ }^{18}$

\section{CONCLUSION}

Peak position of the Faraday rotation spectrum of magnetite/PMMA nanocomposite films is shown to be concentration dependent. By increasing the concentration of 8-nm particles in the PMMA matrix, a redshift and broadening of the main spectral feature are observed. Calculations using existing EMT models fail to account for the shifts seen in the experimental data. Using a DDA-based model, the shifts in peak position are shown to occur based purely on optical effects where only the interparticle distance is varied. In addition, the peak positions of the calculated spectra depend on the geometrical configuration. In future work, we will extend our calculations to include clusters of randomly distributed particles to explain more quantitatively our experimental data.

\section{ACKNOWLEDGMENTS}

The authors acknowledge support from the National Science Foundation EPSCoR Program and the Louisiana State Board of Regents.

${ }^{1}$ S. A. Maier et al., Adv. Mater. (Weinheim, Ger.) 13, 1501 (2001).

${ }^{2}$ L. A. Kelly et al., J. Phys. Chem. B 107, 668 (2003).

${ }^{3}$ M. L. Brongersma et al., Phys. Rev. B 62, R16356 (2000).

${ }^{4}$ A. Yasumori et al., J. Sol-Gel Sci. Technol. 18, 249 (2000).

${ }^{5}$ H. W. Davies and J. P. Llewllyn, J. Phys. D 13, 2327 (1980).

${ }^{6}$ Yu. A. Barnakov et al., J. Phys. Chem. Solids 65, 1005 (2004).

${ }^{7}$ B. T. Draine, Astrophys. J. 333, 848 (1988).

${ }^{8}$ B. T. Draine and P. J. Flatau, J. Opt. Soc. Am. A 11, 1491 (1994).

${ }^{9}$ T. K. Xia, P. M. Hui, and D. Stroud, J. Appl. Phys. 67, 2736 (1990).

${ }^{10}$ R. M. A. Azzam and N. M. Bashara, Ellipsometry and Polarized Light (Elsevier Science, New York 1999).

${ }^{11}$ K. Urano and M. Inoue, J. Chem. Phys. 66, 791 (1977).

${ }^{12}$ M. Abe and M. Gomi, Jpn. J. Appl. Phys., Part 1 23, 1580 (1984).

${ }^{13}$ A. Schlegel, S. F. Alvarado, and P. Wachter, J. Phys. C 12, 1157 (1978).

${ }^{14}$ X. Zhang, J. Schoenes, and P. Wachter, Solid State Commun. 39, 189 (1981).

${ }^{15}$ D. Caruntu et al., Inorg. Chem. 41, 6137 (2002).

${ }^{16}$ K. Sato, Jpn. J. Appl. Phys. 20, 2403 (1981).

${ }^{17}$ V. A. Podolskiy, Opt. Express 11, 735 (2003).

${ }^{18}$ V. A. Markel, J. Mod. Opt. 39, 853 (1992). 